Communications in Physics, Vol. 29, No. 2 (2019), pp. 129-139

DOI:10.15625/0868-3166/29/2/13670

\title{
SAMPLING THE FOLDING TRANSITION STATE ENSEMBLE IN A TUBE-LIKE MODEL OF PROTEIN
}

\author{
NGUYEN BA HUNG ${ }^{1}$ AND TRINH XUAN HOANG ${ }^{2,3, \dagger}$ \\ ${ }^{1}$ Vietnam Military Medical University, 160 Phung Hung, Ha Dong, Hanoi, Vietnam \\ ${ }^{2}$ Institute of Physics, Vietnam Academy of Science and Technology, 10 Dao Tan, Ba Dinh, Hanoi, \\ Vietnam \\ ${ }^{3}$ Graduate University of Science and Technology, Vietnam Academy of Science and Technology, \\ 18 Hoang Quoc Viet, Cau Giay, Hanoi, Vietnam \\ ${ }^{\dagger}$ E-mail: hoang@iop.vast.ac.vn
}

Received 8 March 2019

Accepted for publication 2 May 2019

Published 15 May 2019

\begin{abstract}
We used the tube model with Go-like potential for native contacts to study the folding transition of a designed three-helix bundle and a designed protein G-like structure. It is shown that both proteins in this model are two-state folders with a cooperative folding transition coincided with the collapse transition. We defined the transition states as protein conformations in a small region around the saddle point on a free energy surface with the energy and the conformational root mean square deviation (rmsd) from the native state as the coordinates. The transition state region on the free energy surface then was sampled by using umbrella sampling technique. We show that the transition state ensemble is broad consisting of different conformations that have different folded and unfolded elements.
\end{abstract}

Keywords: free energy landscape, transition state, Monte Carlo simulation.

Classification numbers: 87.15.M-; 87.64.K-.

\section{INTRODUCTION}

Understanding the nature of the transition state, a half-way conformation supposed to be at the maximum of a free energy barrier separating the unfolded (denatured) state and the native state, is crucial for understanding the folding mechanism of two-state proteins. There have been two different views on the folding process. The old view dated back to the late 1960s suggested that folding proceeds through one or a few confined pathways so that the Levinthal paradox can be (C)2019 Vietnam Academy of Science and Technology 
avoided [1]. The new view, arisen in the early 1990s from the energy landscape theory along with the concept of a folding funnel $[2,3]$, described the folding process as a progressive rearrangement of an ensemble of conformations towards lower internal energy and lower conformational entropy. The new view is thus associated with multiple pathways. In the old view, the transition state corresponds to one or a few conformations and the free energy barrier leading to the transition state from the unfolded state would be purely enthalpic. In the new view, the transition states are an ensemble of conformations and the associated free energy barrier was suggested to be primarily entropic $[4,5]$.

Protein engineering method combined with equilibrium and kinetic experiments can provide information about the transition state structure. Such information includes the $\Phi$-values [6], which reveal the degree of native structure around a mutated residue in the transition state. $\Phi=0$ means that the residue does not form a native-like structure in the transition state. $\Phi=1$ means that the structure around the residue in the transition state is the same as in the native state. Fractional $\Phi$-value however indicates that either the residue forms a partial native-like structure in the transition state or the transition state ensemble is a mixture of conformations in which full nativelike structures form in different regions among different conformations. Fersht and coworkers have shown that Chymotrypsin Inhibitor 2 (CI2) has fractional $\Phi$-values and the transition state has no region forming a full native-like structure [7,8]. CI2 was suggested to have a single transition state which corresponds to an ensemble of similar structures resembling an expanded version of the native state. Ubiquitin [9] was reported to have polarized transition state in which some elements of the native structure are fully formed and some other elements are fully unstructured. The SH3 domain [10] was shown to have a defined and conformationally restricted transition state with a $\beta$-turn formed even before the formation of the transition state. These experimental results indicated that proteins fold via multiple pathways, but the pathways are related.

Structure-based Go-like models have been useful for understanding the folding mechanism, and successful in capturing the folding events $[11,12]$ and the folding transition state $[13,14]$ with significant agreement to experiments. In the present study, we attempt to identify the transition state for proteins in a different but related model, the tube model with Go-like potentials for the native contacts, namely the tube Go model. This model inherits both the geometrical constraints and hydrogen bondings from the tube model and also the native structure selectivity from the Golike model. The tube Go model thus is more realistic than Go-like models. We also introduce a new quantity $f$ measuring the fraction of native contacts formed by a residue in the transition state, which is similar to the $\Phi$-value, for the analysis of the transition state structures.

\section{METHODS}

\section{The tube Go model}

We considered the tube model with Go-like potential for native contacts and called it the tube Go model [15]. The latter is exactly the same as the tube model [16] except that the hydrophobic interaction is replaced by the structure-based Go-like interaction [17]. The model assumes the polypeptide chain as a chain of $\mathrm{C}_{\alpha}$ atoms representing amino acid residues located along the axis

of a self-avoiding tube of the thickness radius $\Delta=2.5 \AA$. The tube self-avoidance is enforced by applying a three-body potential, in such a way that the radius of a circle going through any triplet of $\mathrm{C}_{\alpha}$ atoms must be larger than $\Delta$ [18]. A bending energy penalty of constant magnitude $e_{R}=0.3$ 
along the chain is given to any non-terminal residue, at whose position the chain local curvature radius is smaller than $3.2 \AA$. The energetics and geometry of hydrogen bonds are encapsulated in the model based on a statistical analysis of protein native structures [19]. The local (nonlocal) hydrogen-bond energies are defined to be $-1(-0.7)$ with a cooperative energy of -0.3 assigned to any pair of consecutive hydrogen bonds either in a $\beta$-sheet or an $\alpha$-helix.

A Go-like potential is assigned to non-local contacts between the $\mathrm{C}_{\alpha}$ atoms. In a given protein conformation, a contact between two atoms is formed if the distance between them is less than $7.5 \AA$. Non-local contacts are formed by atoms separated by at least 3 amino acids along the chain. A negative energy $e_{W}$ is assigned equally to all non-local native contacts, i.e. the contacts that are present in the protein native state. Non-native contacts are given zero energy.

In the present study, we consider two proteins with the native structures shown in Fig. 1. They are a three-helix bundle denoted as 3HB and a protein G-like structure denoted as GB1. These structures are the ground states of previously designed proteins in the tube model with hydrophobic-polar (HP) sequences [20]. Here, these structures are used as input for determining the native contacts for the Go-like interactions. The energy parameter $e_{W}$ in the Go-like potential for each protein was chosen such that the total energy of all native contacts in the native state is equal to the total hydrophobic energy in the tube model with HP sequences. For protein $3 \mathrm{HB}$, we obtained $e_{W}=-0.229$, and for GB1, $e_{W}=-0.15$. Both proteins have the same chain length with $N=48$ amino acids.

(a)

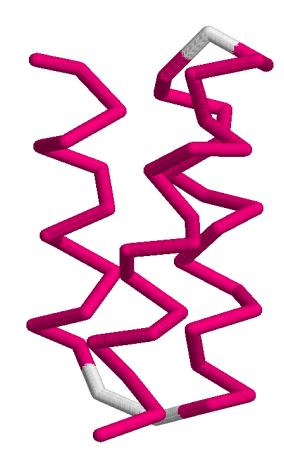

3HB (b)

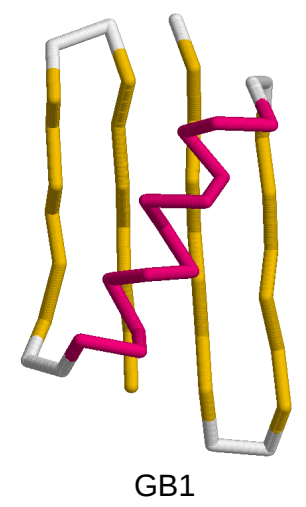

Fig. 1. (a) Structure of a three-helix bundle (3HB). (b) A structure akin to the B1 domain of protein $\mathrm{G}(\mathrm{GB} 1)$. $\alpha$-helices are shown in red color whereas $\beta$-sheets in yellow color.

Parallel tempering [21] Monte Carlo simulations were carried out with the standard pivot and crankshaft moves commonly used in stochastic chain dynamics. The Metropolis algorithm for move acceptance/rejection is used with a thermal weight $\exp (-E / T)$ for each conformation, where $E$ is the energy of the conformation and $T$ is an effective temperature. We have adopted dimensionless units for both energy and temperature with the Boltzmann constant $k_{B}=1$. The weighted histogram method [22] was used to calculate the equilibrium properties such as the 
average energy, the mean radius of gyration, and the specific heat. The latter is defined as

$$
C=\frac{\left\langle E^{2}\right\rangle-\langle E\rangle^{2}}{T^{2}}
$$

The folding temperature $T_{f}$ is defined as the temperature of the maximum of the specific heat.

The radius of gyration of a protein conformation is defined as

$$
R_{g}=\sqrt{\sum_{i=1}^{N}\left(\mathbf{r}_{i}-\mathbf{r}_{\mathrm{cm}}\right)^{2}},
$$

where $\mathbf{r}_{i}$ is the position of residue $i$ and $\mathbf{r}_{\mathrm{cm}}$ is the position of the center of mass of the protein. The root mean square deviation (rmsd) of a conformation to the native state is given by

$$
\mathrm{rmsd}=\sqrt{\min \sum_{i=1}^{N}\left(\mathbf{r}_{i}-\mathbf{r}_{i}^{0}\right)^{2}},
$$

where $\mathbf{r}_{i}^{0}$ is the position of residue $i$ in the native state.

\section{Sampling the transition state ensemble}

To determine the transition state of protein we considered a two-dimensional free energy surface defined as

$$
F(E, \text { rmsd })=-T \log P(E, \text { rmsd }),
$$

where $P(E$, rmsd $)$ is the probability of observing a conformation of energy $E$ and a given rmsd to the native state. The probability $P$ can be determined from equilibrium simulations. The transition state is defined by the saddle point in the free energy surface, presumably with coordinates $\left(E_{0}, \mathrm{rmsd}_{0}\right)$. Due to discretization procedure as well as numerical uncertainty, in practice we assumed the transition state to be found anywhere in a small region around the saddle point, i.e. within $\left(E_{0} \pm 1, \operatorname{rmsd}_{0} \pm 0.5\right)$, where the rmsd is given in units of $\AA$.

Because the transition state is transient during equilibrium simulation, we have employed umbrella sampling technique [23] to effective sample protein conformations in the transition state region, with the use of a restraint potential for the energy $E$

$$
V_{0}(E)=k_{0}\left(E-E_{0}\right)^{2},
$$

where $k_{0}$ is a constant. We have checked that it is good enough to choose $k_{0}=0.01$. In the umbrella sampling simulation, the conformations were sampled with the probability weight

$$
w(E)=\exp \left(-\frac{E+V_{0}(E)}{T}\right) .
$$

In order to characterize the transition state, we defined the quantity $f_{i}$ of each residue position $i$ in the protein chain as

$$
f_{i}=\frac{k_{\ddagger}(i)}{k_{N}(i)},
$$

where $k_{\ddagger}(\mathrm{i})$ is the number of native contacts formed by residue $i$ in the transition state $(\ddagger)$ and $k_{N}(i)$ is the corresponding number in the native state $(\mathrm{N})$. Thus, $f_{i}$ is the fraction of native contacts 
formed by residue $i$ in the transition state. It receives values between 0 and 1 and plays a role similar to that of the $\phi$-value considered in protein engineering experiments. The values of $k_{\ddagger}(i)$ were calculated from umbrella sampling simulations at $T=T_{f}$ as [23]

$$
k_{\ddagger}(i)=\frac{\sum_{\text {TSregion }} k(i)(w(E))^{-1}}{\sum_{\text {TSregion }}(w(E))^{-1}},
$$

where $k(i)$ is the number of native contacts of residue $i$ in a conformation and the summation is taken over all conformations occurred in the transition state region during the simulation. The weights $(w(E))^{-1}$ remove the sampling bias provided by the energy and the restraint potential.

\section{RESULTS AND DISCUSSION}
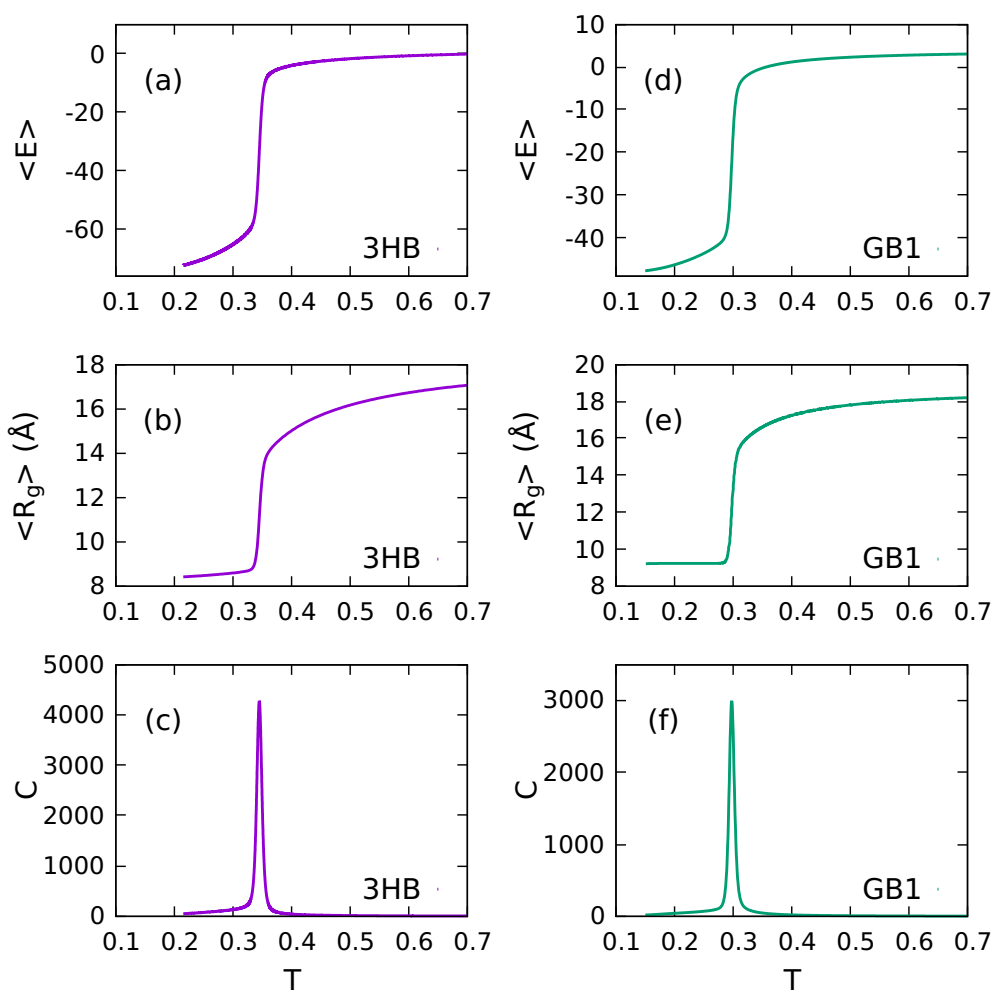

Fig. 2. Temperature dependence of the average energy $(a, d)$, the mean radius of gyration (b,e) and the specific heat (c,f) for protein 3HB (left panels) and protein GB1 (right panels) in the tube Go model.

Figure 2 shows the temperature dependences of the average energy $\langle E\rangle$, the mean radius of gyration $\left\langle R_{g}\right\rangle$, and the specific heat for protein $3 \mathrm{HB}$ and protein GB1. Both the average energy and the mean radius of gyration show a sharp change near a temperature, at which the specific heat $C$ has a very strong and narrow peak. All these behaviors indicate a cooperative folding transition. In a cooperative folding, all parts of the protein fold almost simultaneously without intermediates 
leading to sharp changes of energy and other properties of the molecule at the transition temperature. Our results here also show that the collapse transition, associated with the change in $R_{g}$, happens almost at the same temperature as the folding transition. The folding temperature $T_{f}$, corresponding to the temperature of the maximum of specific heat, was found to be equal to 0.34 for $3 \mathrm{HB}$ and 0.297 for GB1.

Figures 3 and 4 show the time dependences of the energy, the rmsd and the radius of gyration $R_{g}$ in a long simulation at the folding temperature $T_{f}$ for two proteins $3 \mathrm{HB}$ and GB1, respectively. For both proteins, the simulations show multiple switching between conformations of high and low values of the considered quantities, suggesting that the proteins in our model are two-state folders. The conformations of low energy, low rmsd, and low $R_{g}$ correspond to the folded state whereas those of high energy, high rmsd, and high $R_{g}$ correspond to the unfolded state. The histograms of energy, rmsd and $R_{g}$ shown on the right panels of Figs. 3 and 4 also indicate two peaks corresponding to these two states. The separation of the peaks however is more clearly for energy and rmsd than for $R_{g}$, suggesting that energy and rmsd better coordinates for folding than $R_{g}$.
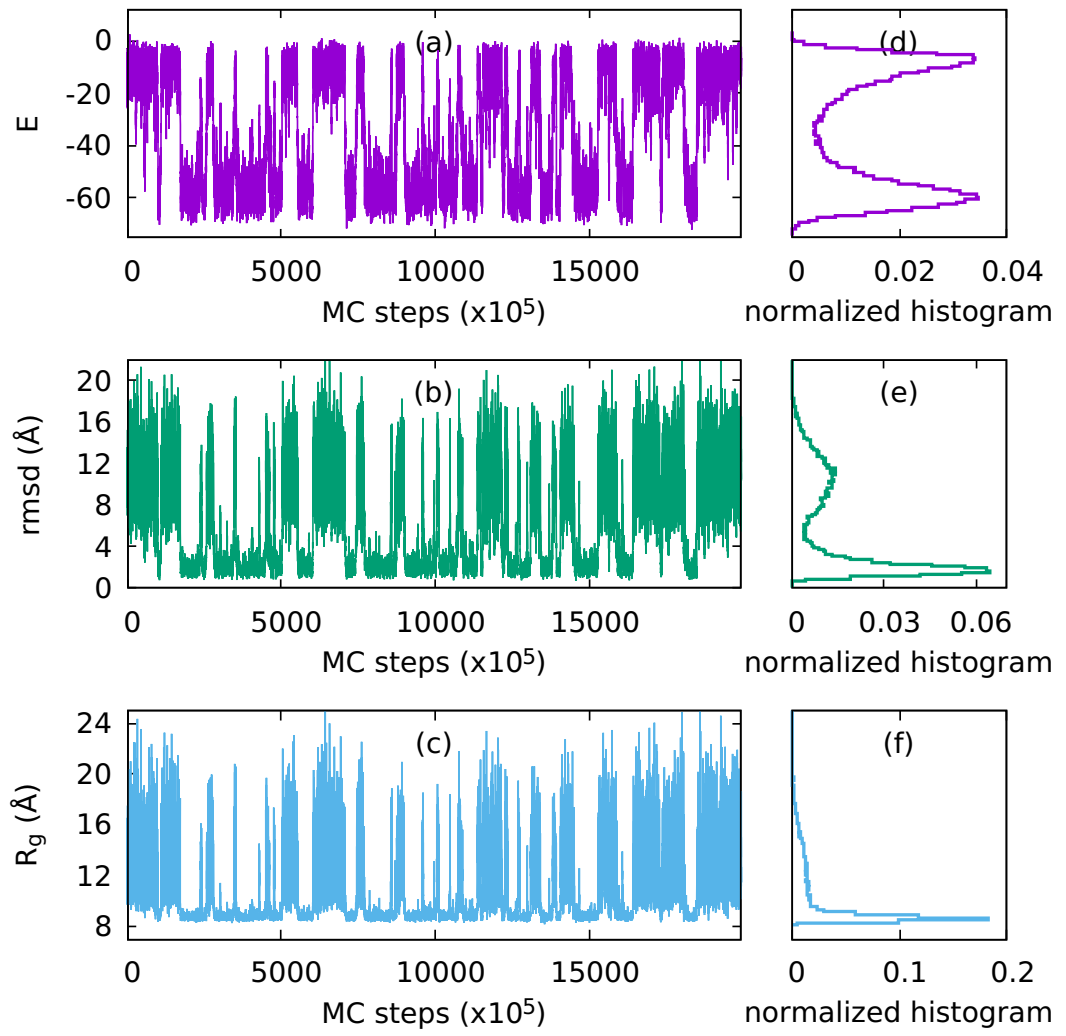

Fig. 3. Time dependence of the energy $E$ (a), the root mean square deviation rmsd (b) and the radius of gyration $R_{g}$ (c) in a long trajectory for protein $3 \mathrm{HB}$ at its folding temperature $T_{f}=0.34$. Time is given in Monte Carlo steps. Right panels show histograms of the quantities considered in the left panels. 

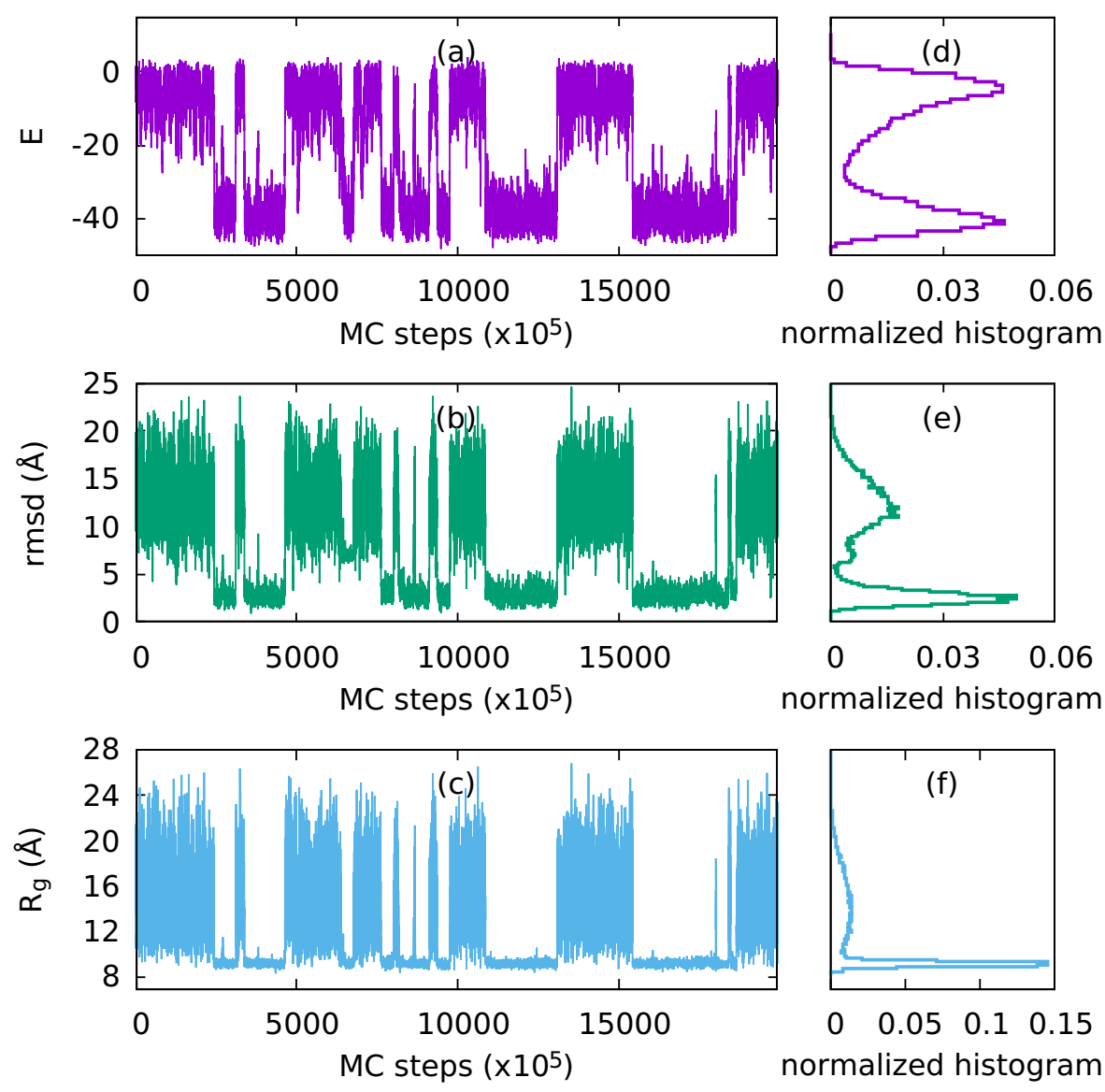

Fig. 4. Same as Fig. 3 but for protein GB1 at its folding temperature $T_{f}=0.297$.

Figure 5 shows the dependence of the free energy on the number of native contacts for the two proteins $3 \mathrm{HB}$ and GB1 at their folding temperature $T_{f}$. For both proteins, the free energy has two minima corresponding to the unfolded state and the folded state separated by a barrier. The folding and unfolding free energy barriers are found to be in the range from 0.6 to 1 in units of local hydrogen bond energy, or equivalently $3-5 k_{B} T$. These low free energy barriers indicate that the modeled proteins are fast folders.

Figure 6 shows contour plots of the free energy surface $F(E$,rmsd) along the two coordinates $E$ and rmsd for protein $3 \mathrm{HB}$ and protein GB1. The free energy was obtained from long simulations at $T=T_{f}$. For both proteins, the free energy surface has two minima, one corresponds to the folded state at low $E$ and low rmsd and the other one corresponds to the unfolded state at high $E$ and high rmsd. There is a saddle point between the two minima supposed to be the position of the transition state on the free energy surface. The coordinates $\left(E_{0}, \operatorname{rmsd}_{0}\right)$ of the saddle point on the surface can be approximately read from the contour plots as $(-23,6 \AA)$ and $(-18,8 \AA)$ for $3 \mathrm{HB}$ and GB1, respectively. 


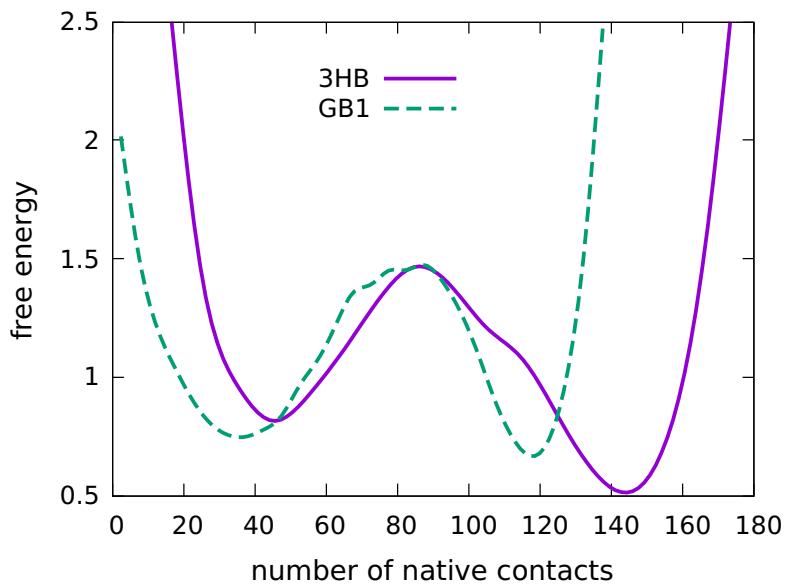

Fig. 5. Dependence of the free energy on the number of native contacts for $3 \mathrm{HB}$ (solid) and GB1 (dashed) at their folding temperatures $T_{f}$. The free energy was obtained as $F(Q)=-T \log P(Q)$, where $P(Q)$ is the probability of observing conformations of the number of native contacts $Q$ in equilibrium simulations.

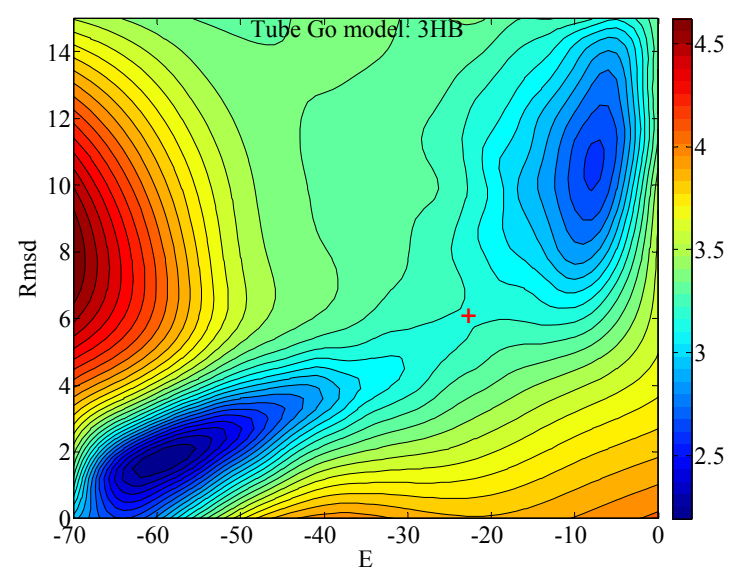

(a)

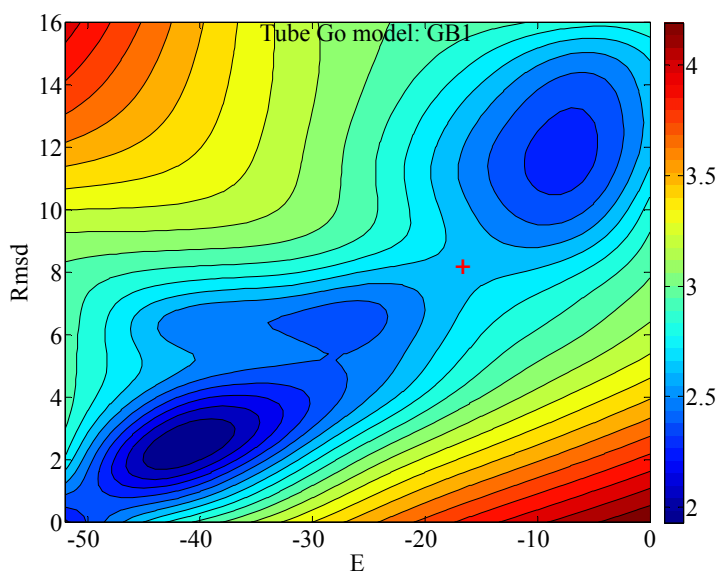

(b)

Fig. 6. Contour plots of the free energy as function of the energy $E$ and the rmsd for protein 3HB (a) and protein GB1 (b) at their folding temperatures $T_{f}$. The approximate position of the transition state at the saddle point is indicated by the cross.

We have carried out umbrella sampling simulations to sample protein conformations in the transition state region as described in the Methods section. With a run of $4 \times 10^{9} \mathrm{MC}$ steps and the sampling frequency of one conformation every $10^{4}$ steps we were able to obtain about 1000 conformations in the transition state region. Figure 6 shows the values of $f_{i}$ obtained for all amino acid positions in the two proteins and some examples of transition state conformations found near the saddle points. For protein $3 \mathrm{HB}, f_{i}$ varies mainly between 0.2 and 0.5 with some 
values slightly larger than 0.5 for a few positions around the middle of the protein sequence. This profile of $f_{i}$ indicates that all the positions in the sequence of the $3 \mathrm{HB}$ protein mildly contribute to the formation of the transition state though not equally. The values of $f_{i}$ found to be substantially less than 1 indicate that the transition state ensemble includes a variety of different conformations of different sets of native contacts. In other words, one can say that the transition state is broad. In consistency with the calculated $f_{i}$ values, the transition state conformations shown in the bottom of Fig. 7a are very different from each other thought they have almost the same energy and the same rmsd with respect to the native state. One can notice that the transition state may have one or two pieces of the $\alpha$-helices formed and the rest of the conformation disordered. We have checked that $\beta$-sheets typically are not present in the transition state ensemble of $3 \mathrm{HB}$. However, in less than $8 \%$ of the snapshots in the TS ensemble, some small amount of $\beta$ structure (4-8 residues) can be observed.
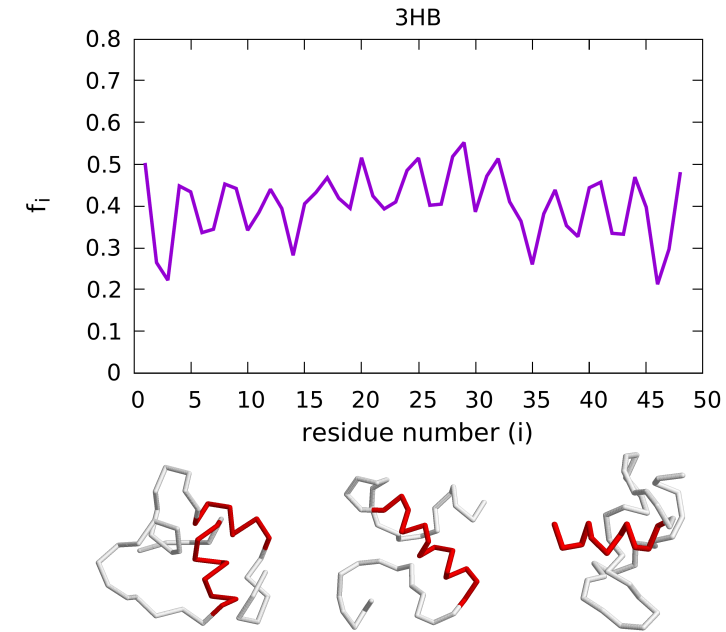

$E=-23.259$ $\mathrm{rmsd}=5.990 \AA$

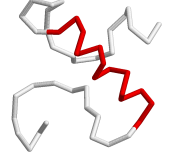

$E=-23.001$ rmsd $=5.989 \AA$

(a)
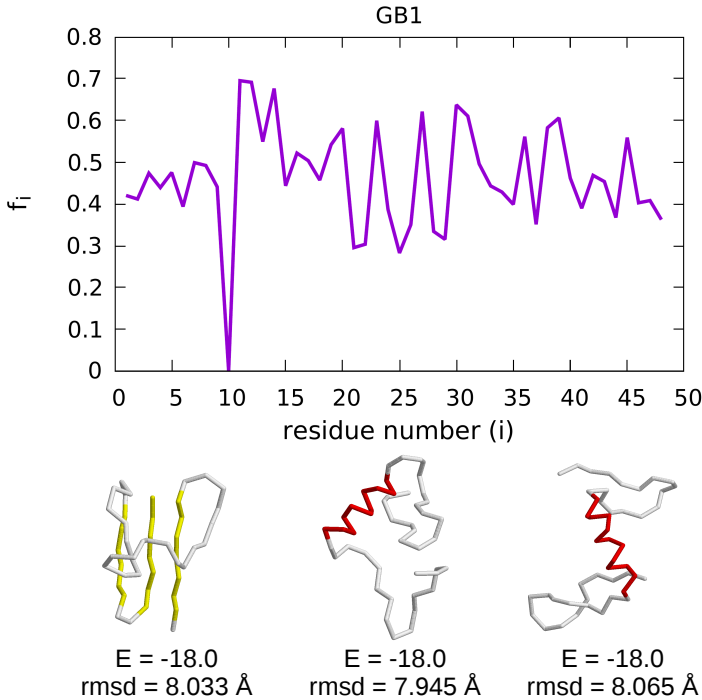

(b)

Fig. 7. (a) (Top) Dependence of the quantity $f_{i}$ on the residue number $i$ for protein $3 \mathrm{HB}$. (Bottom) Examples of transition state conformations for protein 3HB with the corresponding values of energy and rmsd as indicated. (b) Same as (a) but for protein GB1. Secondary structures formed in the transition state are shown in colors.

The $f_{i}$ profile for protein GB1 shows stronger variation than for $3 \mathrm{HB}$, with the values range mostly from 0.3 to 0.7 . At position $i=10$, the value $f_{i}=0$ is shown because the residue number 10 has no native contacts. Some values of $f_{i}$ for GB1 are somewhat higher than those of $3 \mathrm{HB}$, but the conclusions about the transition state are similar for the two cases. For GB1, the transition state is also broad with a large variety of conformations. The examples of conformations shown in Fig. $7 \mathrm{~b}$ indicate that the transition state may arise with the formation of a portion of the $\beta$-sheet or a piece of the $\alpha$-helix, whereas the rest of the conformation is largely disordered.

There has been a rich body of theoretical studies on the folding mechanism of proteins with native state topologies similar to those considered in our study. The 46-residue three-helix bundle 
of the B domain of staphylococcal protein A was studied by Zhou and Karplus [24,25] in a Go-like model. They showed that thermodynamically this protein has multiple transitions between various phases of different degrees of ordering, while kinetically it may have on-pathway and off-pathway intermediates. A folding intermediate of this three-helix bundle domain was also found in a free energy calculation by Boczko and Brooks [26] and in a kinetic study by Yang et al. [27] via allatom simulations. Shimada and Shakhnovich [28] studied the folding of the 57-residue B1 domain of protein $\mathrm{G}$ in an all-atom model with Go-like potential and showed that this protein has an onpathway intermediate. A more recent study of Kmiecik and Kolinski [29] using a coarse-grained model with statistical potential also indicated that protein $\mathrm{G}$ folds via three-state mechanism with a molten globule intermediate. In contrast to the above theoretical studies, our tube Go model shows two-state behavior with a single sharp transition between the unfolded phase and the folded phase for both the $3 \mathrm{HB}$ and the GB1 proteins.

Experimentally, protein A was shown to have rapid two-state kinetics with no evidence of intermediates [30-32], whereas whether protein $\mathrm{G}$ is a two-state or three-state folder is under debate $[33,34]$. The behaviors of $3 \mathrm{HB}$ and GB1 thus are partially in agreement with experiment. The experiments also reported high $\Phi$-values $(\approx 0.8-1)$ for some residues in the helix 1 and helix 2 of protein A [31] and in the second $\beta$-turn of protein G [34]. Our $f$ values obtained for $3 \mathrm{HB}$ and GB1 do not reflect these experimental results. Note that the native structures of $3 \mathrm{HB}$ and GB1 are designed structures taken from previous study of the tube model and thus to some extents are different from real structures of protein $\mathrm{A}$ and protein $\mathrm{G}$. The designed proteins and real proteins are also different in chain length. Thus, our study only serves as a general consideration of twostate proteins but not for a detailed comparison with experiment for specific proteins.

\section{CONCLUSION}

Our study comes with two main findings. The first finding is that the tube Go model yields cooperative two-state folding characteristic similar to that of small globular proteins. This characteristic arises from both the geometrical and energetic components of the tube model, which are associated with basic properties of a polypeptide chain, and the native-centric potentials of the Go model accounting for the effect of the amino acid sequence. The tube model yields a presculpted free energy landscape [16] with few energy minima while the Go-like potential provides the selectivity of the native state as the global minimum. As a more realistic feature than standard Go-like models, the tube Go model can form non-native hydrogen bonds which compete with native interactions. Our results show that this competition does not destroy the two-state folding behavior. The second finding is that the transition state ensemble of protein is broad consisting of largely different conformations, which indicates that proteins fold through multiple independent or weakly related parallel pathways. This scenario is fully consistent with the new view of protein folding, while experiments seem to indicate more strongly related pathways. Further investigations are needed to understand this gap between theory and experiment.

\section{ACKNOWLEDGMENTS}

This research is funded by Vietnam National Foundation for Science and Technology Development (NAFOSTED) under grant number 103.01-2016.61. 


\section{REFERENCES}

[1] C. Levinthal, J. Chim. Phys. 65 (1968) 44.

[2] P. E. Leopold, M. Montal and J. N. Onuchic, Proc. Natl. Acad. Sci. USA 89 (1992) 8721.

[3] P. G. Wolynes, J. N. Onuchic and D. Thirumalai, Science 267 (1995) 1619.

[4] A. Šali, E. Shakhnovich and M. Karplus, J. Mol. Biol. 235 (1994) 1614.

[5] J. N. Onuchic, N. D. Socci, Z. Luthey-Schulten and P. G. Wolynes, Fold. Des. 1 (1996) 441.

[6] A. R. Fersht, A. Matouschek and L. Serrano, J. Mol. Biol. 224 (1992) 771.

[7] D. E. Otzen, L. S. Itzhaki, S. Jackson and A. Fersht, Proc. Natl. Acad. Sci. USA 91 (1994) 10422.

[8] A. R. Fersht, L. S. Itzhaki, J. Matthews and D. Otzen, Proc. Natl. Acad. Sci. USA 91 (1994) 10426.

[9] H. M. Went and S. E. Jackson, Prot. Eng. Des. Sel. 18 (2005) 229.

[10] J. C. Martinez, M. T. Pisabarro and L. Serrano, Nat. Struct. Mol. Biol. 5 (1998) 721.

[11] T. X. Hoang and M. Cieplak, J. Chem. Phys. 112 (2000) 6851.

[12] T. X. Hoang and M. Cieplak, J. Chem. Phys. 113 (2000) 8319.

[13] C. Clementi, H. Nymeyer and J. N. Onuchic, J. Mol. Biol. 298 (2000) 937.

[14] C. Clementi, A. E. Garcia and J. N. Onuchic, J. Mol. Biol. 326 (2003) 933.

[15] N. B. Hung and T. X. Hoang, Comm. Phys. 23 (2013) 313.

[16] T. X. Hoang, A. Trovato, F. Seno, J. R. Banavar and A. Maritan, Proc. Natl. Acad. Sci. USA 101 (2004) 7960.

[17] N. Go, Ann. Rev. Biophys. Bioeng. 12 (1983) 183.

[18] A. Maritan, C. Micheletti, A. Trovato and J. R. Banavar, Nature (London) 406 (2000) 287.

[19] J. R. Banavar, T. X. Hoang, A. Maritan, F. Seno and A. Trovato, Phys. Rev. E 70 (2004) 041905.

[20] T. X. Hoang, L. Marsella, A. Trovato, F. Seno, J. R. Banavar and A. Maritan, Proc. Natl. Acad. Sci. USA 103 (2006) 6883 .

[21] R. H. Swendsen and J.-S. Wang, Phys. Rev. Lett. 57 (1986) 2607.

[22] A. M. Ferrenberg and R. H. Swendsen, Phys. Rev. Lett. 63 (1989) 1195.

[23] G. M. Torrie and J. P. Valleau, J. Comp. Phys. 23 (1977) 187.

[24] Y. Zhou and M. Karplus, Proc. Natl. Acad. Sci. USA 94 (1997) 14429.

[25] Y. Zhou and M. Karplus, Nature 401 (1999) 400.

[26] E. M. Boczko and C. L. Brooks, Science 269 (1995) 393.

[27] J. S. Yang, S. Wallin and E. I. Shakhnovich, Proc. Natl. Acad. Sci. USA 105 (2008) 895.

[28] J. Shimada and E. I. Shakhnovich, Proc. Natl. Acad. Sci. USA 99 (2002) 11175.

[29] S. Kmiecik and A. Kolinski, Biophys. J. 94 (2008) 726.

[30] J. K. Myers and T. G. Oas, Nat. Struct. Mol. Biol. 8 (2001) 552.

[31] S. Sato, T. L. Religa, V. Daggett and A. R. Fersht, Proc. Natl. Acad. Sci. USA 101 (2004) 6952.

[32] S. Sato, T. L. Religa and A. R. Fersht, J. Mol. Biol. 360 (2006) 850.

[33] S.-H. Park, M. R. Shastry and H. Roder, Nat. Struct. Mol. Biol. 6 (1999) 943.

[34] E. L. McCallister, E. Alm and D. Baker, Nat. Struct. Mol. Biol. 7 (2000) 669. 